\title{
Perfil dos usuários de substâncias psicoativas de um Hospital Universitário do Rio Grande do Sul
}

\author{
Natalia Dalla Côrte Cantarelli' Elsa Karsburg da Rosa Marchesan² Marion Caroline do Amaral² Jadir Camargo Lemos ${ }^{3}$
}

\begin{abstract}
RESUMO
O presente estudo buscou conhecer o perfil dos usuários de substâncias psicoativas que internaram no período de outubro de 2009 a outubro de 2010 em um Hospital Universitário do Rio Grande do Sul. A pesquisa caracterizou-se como exploratória documental, de natureza quantitativa, através de uma busca nos registros de 177 prontuários. $O$ instrumento da pesquisa foi constituído por questões referentes a informações sociodemográficas, histórico de internações e dados referentes à condição de saúde. Constatou-se um considerável número de internações por homens, entre 31 e 50 anos, sem ocupação e uso prevalente de álcool em tempo médio de 24 anos - dado que sinaliza que não só o contato inicial, mas também, o abuso do consumo iniciou na adolescência. Considera-se que este estudo pode contribuir para a ampliação do conhecimento sobre o fenômeno das drogas, fato que poderá auxiliar o desenvolvimento de práticas de cuidado adequadas a essa população.
\end{abstract}

Descritores: Abuso de Substâncias, Adolescência, Saúde Pública.

\section{Profile the psychoactive substances users of a University Hospital of Rio Grande do Sul}

\begin{abstract}
This study aims to recognize the psychoactive substances users profile that hospitalized from October 2009 to October 2010 at the University Hospital of Rio Grande do Sul. The research was documental exploratory (quantitative) for a total sample amount of 177 medical records for the period considered. During the research questions regards to sociodemographic information, hospitalization history and health data were considered. As the result, it may be observed that the majority number of hospitalized people are men between 31 and 50 years old, with a mean of 24 years of alcohol use, what indicate that this people start to consume alcohol in its adolescence. This alcohol consume when constant and abusive cause disturbances in the social, work and family relationships. This study may contribute in the development of new health practices to threat alcohol diseases.
\end{abstract}

Descriptors: Substance-Related Disorders, Public Health, Adolescence.

\footnotetext{
${ }^{1}$ Mestranda em Psicologia na Universidade Federal de Santa Maria (UFSM), Santa Maria, RS, Brasil.

${ }^{2}$ Especialização em Residência Multiprofissional Integrada pela Universidade Federal de Santa Maria (UFSM), Santa Maria, RS, Brasil.

${ }^{3}$ Doutor em Engenharia de Produção pela Universidade Federal de Santa Catarina (UFSC), Florianópolis, SC, Brasil.
} 


\section{Introdução}

O fenômeno das drogas tem sido amplamente divulgado e discutido em nossa sociedade, uma vez que tem se constituído como um problema de grande complexidade. Através do reconhecimento da relação entre o uso de substâncias psicoativas e inúmeros agravos sociais decorrentes, a toxicomania passou a ser compreendida como uma séria questão social e de saúde pública'.

Durante muito tempo, o olhar voltado para os usuários de drogas esteve orientado por políticas que se restringiam ao combate do uso e do comércio dessas substâncias, com o intuito de preservar a segurança do país. Essa forma de abordagem, que data do início do século XX, pautava-se na crença de que os usuários eram um perigo para a ordem moral vigente e deviam ser identificados, perseguidos e tratados em reclusão².

Diante desse contexto, as iniciativas de práticas de saúde desenvolveram-se em meio a uma sociedade que via o usuário como um perigo para o bem-estar social. Consequentemente, essas ações acabaram sendo orientadas por práticas excludentes, padronizadas e que não levavam em consideração as necessidades dos usuários ${ }^{3}$.

Por volta da metade do século XIX, através da constatação da correlação entre o uso de substâncias psicoativas e agravos sociais, principalmente nas esferas afetiva, educativa, produtiva, econômica, de saúde e de relações sociais; tornou-se necessário pensar em mudanças nas formas de abordar a toxicomania. Passou a ser imprescindível considerar esta problemática em relação a questões associadas ao campo da saúde através de uma compreensão global do problema, e levando-se em conta a condição biopsicossocial do usuário4.

No entanto, embora a percepção em torno da realidade do uso de álcool e outras drogas tenha se transformado, ainda hoje, evidenciam-se lacunas nos programas e práticas de saúde. Exemplo disso são abordagens terapêuticas que ainda estão pautadas pelo combate às substâncias, principalmente às ilícitas, o que sugere resquícios do olhar punitivo do início do século XX5.

Em relação à importância acerca do conhecimento sobre o perfil dos usuários, um dado importante encontra-se na observação de Reis, Silva ${ }^{6}$ sobre o aumento do uso de drogas entre os adolescentes nas últimas décadas. No entanto, este é um dado que ainda não encontra ressonância nas concepções e modelos de abordagens práticas e requer maiores estudos e reflexões ${ }^{7}$.

Diante desse contexto, o presente estudo partiu da necessidade de ampliar o conhecimento em relação ao fenômeno das drogas através de maiores informações sobre o perfil dos usuários de substâncias psicoativas em relação às substâncias que consomem, à média de tempo que fazem uso e à situação ocupacional. Nesse sentido, esta pesquisa teve por objetivo identificar o perfil dos usuários de um serviço de recuperação de dependentes químicos de um Hospital Universitário do Rio Grande do Sul, durante o período de um ano.

\section{Metodologia}

A pesquisa realizada foi do tipo documental, exploratória, transversal e de natureza quantitativa, desenvolvida no período de dezembro de 2010 a fevereiro de 2011 no serviço de recuperação de dependentes químicos de um Hospital Universitário da região centro do Rio Grande do Sul. A coleta dos dados foi feita pelos pesquisadores no Serviço de Arquivo Médico e Estatístico (SAME) do hospital em questão, tendo sido feita uma busca inicial em 177 prontuários, referentes ao registro de internação de usuários que buscaram o serviço no período de outubro de 2009 a outubro de 2010.

A unidade hospitalar, na qual foi realizado o estudo, interna pacientes de ambos os sexos para tratamento de álcool e outras drogas, à exceção de tabaco. Além disso, conta com 15 leitos, sendo três destinados para mulheres. 0 tempo de internação varia em torno de sete dias, havendo avaliação da equipe para definir se o prazo deve ou não se estender.

$\mathrm{O}$ instrumento de pesquisa foi constituído por trinta e seis questões fechadas, organizadas em blocos $\mathrm{A}, \mathrm{B}$ e $\mathrm{C}$, que corresponderam, respectivamente, às informações sóciodemográficas, ao histórico de internações e aos dados referentes à condição de saúde do usuário na última internação. O conteúdo deste instrumento foi estruturado e fundamentado com base nos estudos de Pelisoli, Moreira ${ }^{8}$ e Formiga, Santos, Dumcke, Araujo ${ }^{9}$, sendo que algumas adaptações foram feitas para atender 0 objetivo desta pesquisa. 
Realizou-se um pré-teste do instrumento de pesquisa com 60 prontuários com a finalidade de garantir que a coleta de dados ocorresse de maneira padronizada pelos pesquisadores. Tornou-se possível, a partir disso, identificar possíveis dúvidas que viessem ocorrer ao longo desse processo, o que auxiliou o melhoramento do instrumento.

De acordo com o referido período da amostra, totalizou-se 177 prontuários referentes aos registros de pacientes internados, sendo que 17 foram excluídos da pesquisa, pois não estavam disponíveis no arquivo durante o período de coleta. Disso decorreu uma amostra disponível de 160 prontuários para o período considerado. Os prontuários analisados foram preenchidos pelos profissionais de saúde, atuantes no local, durante esse período de internação do usuário.

O estudo seguiu os preceitos éticos que regulamentam a pesquisa com seres humanos (Resolução 466/12), sendo que a coleta de dados iniciou após pronunciamento favorável da Direção de Ensino, Pesquisa e Extensão do Hospital; e após o parecer favorável do Comitê de Ética, tendo sido aprovado em reunião do dia 13 de dezembro com CAEE 0307.0.243.0001010. Firmou-se, também, o compromisso de que as informações seriam utilizadas, única e exclusivamente, para fins acadêmico-científicos. Tanto a organização quanto a sistematização dos dados foram realizadas através do programa Microsoft Excel, tendo sido analisados estatisticamente de forma descritiva, e os resultados apresentados em tabela em forma de frequência e percentual.

\section{Resultados e Discussão}

Através do processo de sistematização dos dados, constatou-se um elevado número de internações de usuários na faixa etária entre 31 e 50 anos, sendo a grande maioria homens, conforme pode ser observado na Tabela 1. Desses sujeitos, os dados sobre ocupação revelaram que grande parte estava sem trabalho, o que pode ser evidenciado na Tabela 2. Outro ponto relevante, identificado pela pesquisa, diz respeito ao fato de que, através do cruzamento e estruturação das variáveis de sexo, idade e substância de consumo, revelou-se um número expressivo do uso prevalente de álcool.

Tabela 1 - Distribuição dos sujeitos em relação às variáveis demográficas e substância de consumo.

\begin{tabular}{c|c|c|c|c|c|c|c|c|c|c|c}
\hline \multicolumn{10}{c|}{ Alcool } & \multicolumn{1}{c|}{$\begin{array}{c}\text { Alcool + outra } \\
\text { droga }\end{array}$} & \multicolumn{2}{c|}{ Outra droga } & \multicolumn{2}{|c|}{ NC* } & \multicolumn{2}{|c}{ Total } \\
\hline Sexo & Idade & \multicolumn{2}{|c|}{} & & & & & & \\
\hline & & $\mathrm{n}$ & $\%$ & $\mathrm{n}$ & $\%$ & $\mathrm{n}$ & $\%$ & $\mathrm{n}$ & $\%$ & $\mathrm{n}$ & $\%$ \\
\hline Masculino & 17 a 30 anos & 2 & $1,25 \%$ & 8 & $5,00 \%$ & 1 & $0,63 \%$ & 0 & $0 \%$ & 11 & $6,88 \%$ \\
\hline & 31 a 50 anos & 72 & $45 \%$ & 25 & $15,63 \%$ & 0 & $0 \%$ & 0 & $0 \%$ & 97 & $60,63 \%$ \\
\hline & $>50$ anos & 41 & $25,63 \%$ & 2 & $1,25 \%$ & 0 & $0 \%$ & 0 & $0 \%$ & 43 & $26,88 \%$ \\
\hline Feminino & 17 a 30 anos & 0 & $0 \%$ & 0 & $0,00 \%$ & 0 & $0 \%$ & 1 & $0,63 \%$ & 1 & $0,63 \%$ \\
\hline & 31 a 50 anos & 4 & $2,50 \%$ & 2 & $1,25 \%$ & 0 & $0 \%$ & 0 & $0 \%$ & 6 & $3,75 \%$ \\
\hline & $>50$ anos & 2 & $1,25 \%$ & 0 & $0,00 \%$ & 0 & $0 \%$ & 0 & $0 \%$ & 2 & $1,25 \%$ \\
\hline Total & & 121 & $75,63 \%$ & 37 & $23,13 \%$ & 1 & $0,63 \%$ & 1 & $0,63 \%$ & 160 & $100 \%$ \\
\hline
\end{tabular}

Tabela 2 - Distribuição dos sujeitos em relação à situação ocupacional

\begin{tabular}{c|c|c|c|c|c}
\hline \multicolumn{2}{c|}{ Total de sujeitos } & \multicolumn{4}{c}{ Situação ocupacional } \\
\hline & & \multicolumn{3}{|c}{ Sujeitos com ocupação } & \multicolumn{2}{c}{ Sujeitos sem ocupação } \\
\hline $\mathrm{n}$ & $\%$ & $\mathrm{n}$ & $\%$ & $\mathrm{n}$ & $\%$ \\
\hline 160 & $100 \%$ & 71 & $44,56 \%$ & 89 & $55,44 \%$ \\
\hline
\end{tabular}

Considerando os dados identificados na Tabela 1, em relação à substância de consumo, pode-se inferir que, embora exista um número relevante de usuários de sustâncias ilícitas que procuraram internação no referido período, a grande maioria dos sujeitos buscou o serviço devido ao uso abusivo de álcool. Acredita-se que esse resultado merece atenção, pois, atualmente, assiste-se a uma abordagem antidrogas que se fundamenta na repressão implacável às drogas ilícitas, muitas vezes, descritas como as substâncias mais consumidas ou que mais causam dependência. Fato que acaba por ocultar as consequências do abuso causado pelas substâncias lícitas ${ }^{5}$. 
Diante da evidência de que a maior parte dos usuários buscou o serviço de internação pelo uso abusivo de álcool, pode-se pensar na correlação entre o consumo dessa substância e questões sociais de ordem ocupacional evidenciados na pesquisa. Sobre isso, constatou-se um grande número de sujeitos estavam sem ocupação $(55,44 \%)$, conforme explicitado na Tabela 2.

Não é novidade que o abuso de álcool é um dos maiores problemas de saúde devido ao impacto mundial que acarreta; tanto em termos sociais, quanto financeiros ${ }^{11}$. No entanto, este panorama acaba, frequentemente, mascarado pela ênfase que nossa sociedade confere às drogas ilícitas ${ }^{12}$. Percebe-se uma diferenciação de significados que permeia a questão das drogas lícitas e ilícitas e que pode ser facilmente identificada através de qualquer publicitação no universo midiático que verse sobre esta temática.

Caso o leitor queira se aventurar num exercício mental com o intuito de tentar recordar alguma exposição midiática sobre 0 assunto, provavelmente, o conteúdo imagético formado será consoante com a grande contradição de sentidos que envolve as drogas lícitas e ilícitas. De um lado, as lícitas, geralmente abordadas através de uma publicidade sofisticada que estimula o consumo, evocando uma imagem glamourizada, vinculada à saúde, à beleza e ao sucesso. Por outro lado, as drogas ilícitas, em que se vinculam imagens de perigo, de doença e de morte, além da indissociação do sentimento de prazer ${ }^{13}$.

Segundo Bucher, Oliveira ${ }^{5}$, essa desproporção representativa, presente no discurso social, reserva para a questão das drogas um lugar de mito construído em que a narrativa simbólica é usada, muito mais, para combater uma série de desvios da ordem social do que para tratar a questão das drogas em si. Pode-se dizer, então, que as produções publicitárias, inscritas nesse contexto, de certa forma exercem o papel de cúmplices nas explicações e justificações dessa visão preconcebida.

A partir disso, considera-se importante pensar o grande consumo de drogas lícitas, identificados no presente estudo, através do que Mesquita ${ }^{14}$ aponta se tratar de um consumo que é estimulado como produto "com mercado e marketing estabelecidos" na atualidade. 0 autor refere que há uma grande estimulação da comercialização de substâncias lícitas no Brasil que decorrem na dificuldade do país em encontrar um modo culturalmente aceitável de regulamentar a utilização das drogas, no sentido de que seja capaz de evitar o dano à saúde dos usuários e, ao mesmo tempo, o dano à sociedade.

Em outras palavras, a visão reducionista acerca do assunto "drogas" não fica restrita à mídia, mas estende-se a outros âmbitos. Pode-se pensar, inclusive, em suas repercussões nas políticas nacionais de saúde. Sobre isso, em um estudo realizado a partir de uma reflexão teórica sobre as políticas públicas brasileiras que versam sobre a problemática das drogas, Souza, Kantorski ${ }^{7}$ concluíram que as mesmas têm se pautado em diretrizes que acabam por enfatizar a distinção entre as drogas lícitas e ilícitas.

Os autores ressaltam que é a partir desta diferenciação entre as substâncias psicoativas que se configura a premissa de "um ideal de sociedade protegida do uso de drogas ilícitas e uso indevido de drogas lícitas" (p.2). Essa consideração, segundo Souza, Kantorski ${ }^{7}$, abre margem para uma ênfase no caráter protetivo, o qual remete ao significado de periculosidade inerente ao uso de drogas, o qual vem a reforçar a concepção moral de proibição de um comportamento mau/inadequado.

Ainda em relação à substância de consumo, os dados encontrados na presente pesquisa demonstram a necessidade de refletir sobre o fato de que as consequências advindas do abuso da substância atingem não só o sujeito que faz uso, mas, também, os familiares e a sociedade na qual está inserido. Como exemplo disso, tem-se o grande número de sujeitos identificados na pesquisa que estavam sem ocupação (55,44\%). Ressalta-se, aqui, o fato de que a prevalência desses usuários encontra-se na faixa etária de 31 a 50 anos, a qual é identificada como o período de vida que correspondente socialmente ao de maior produtividade do ser humano. A expectativa para esses indivíduos seria, dessa forma, que estivessem em formação escolar, iniciando ou consolidando sua atividade ocupacional. Porém, evidencia-se que mais da metade dos sujeitos dessa faixa etária, ou não iniciaram uma atividade ocupacional, ou perderam o emprego.

As dificuldades observadas em relação às questões ocupacionais podem estar relacionadas ao fato de que o indivíduo, gradualmente, passa a ter suas condições físicas, psicológicas e sociais comprometidas, à medida que o álcool ou outras drogas passam a assumir o lugar de prioridade em sua vida. Somado a isso, não se pode perder de vista o forte estigma social que enfrentam, o que acaba por dificultar as possibilidades de trabalho ou, mesmo, a permanência no emprego ${ }^{15}$.

Outro dado que merece atenção, refere-se ao tempo de uso abusivo da substância por parte desses usuários, o qual corresponde a 24 anos, em média. Esse dado sinaliza que, não só o contato inicial ocorreu na adolescência, mas também, o consumo abusivo se configurou por volta dos 18 anos. 
Pode-se dizer que a adolescência compreende um período crucial do desenvolvimento, caracterizado por intensas mudanças corporais, por conflitos internos e relacionais, o que a torna um difícil processo a ser vivenciado. Trata-se, portanto, de uma fase que guarda profundas modificações e grandes instabilidades, o que poderia estar associado ao envolvimento com situações de vulnerabilidades, como o consumo de substâncias psicoativas ${ }^{16}$.

Entretanto, mesmo que as peculiaridades deste processo possam tornar o indivíduo mais "vulnerável" ao álcool e a outras drogas, entende-se que 0 contato com essas substâncias faz parte de qualquer cultura e pode ocorrer em qualquer faixa etária. Assim, considera-se essencial, antes de tudo, fornecer instrumentos para que o possível contato produza o menor prejuízo ao indivíduo e à sociedade ${ }^{17}$.

Cabe mencionar que, apesar da série de estudos que apontam a adolescência como uma fase de grande exposição ao consumo e possível desenvolvimento do uso abusivo de psicotrópicos, verifica-se a falta de serviços, intervenções, programas e espaços dedicados a esse público. ${ }^{18,19,20,21}$ Nos próprios programas de atenção a usuários de álcool e outras drogas, pode-se evidenciar esse paradoxo. Por um lado, a constatação de que a adolescência constitui um período de fragilidades que tornam o adolescente mais suscetível às drogas. Por outro, textos que regulamentam as políticas públicas e que, embora contemplem a necessidade de desenvolver ações específicas para esse público, não especificam, nem regulam essas práticas.

Constata-se, nestes programas, a carência do reconhecimento de um lugar próprio para o adolescente na construção do cuidado. Acredita-se que a escassez de práticas destinadas à atenção à adolescência pode estar associada ao prolongamento do uso abusivo de substâncias o que sugere a relação com uma série de complicações ao longo da vida adulta, como dificuldades em formar ou manter uma família, atividades ocupacionais, dentre outros.

\section{Conclusão}

Através da identificação do perfil dos usuários de substâncias psicoativas de um Hospital Universitário do Rio Grande do Sul, pode-se evidenciar que a grande maioria dos sujeitos que buscou o serviço de internação, no referido período pesquisado, deu-se em função do consumo prevalente de álcool. Acredita-se que esse fato aponte para a necessidade de reflexões em relação à forma como a questão das drogas tem sido abordada, pois, muitas vezes, observa-se que os problemas em decorrência do consumo de álcool são mascarados pela ênfase que nossa sociedade coloca nas drogas ilícitas.

Além disso, constatou-se que a faixa etária de maior incidência encontra-se entre os 31 e 50 anos, a qual é vista socialmente como a fase de maior produtividade em nossa sociedade. No entanto, uma grande parcela desses sujeitos estava desempregada. Com base nestes dados, pode-se pensar que o prolongamento do uso abusivo das drogas esteja associado a complicações desenvolvidas ao longo da vida adulta, conforme indica o estudo.

Constatou-se, ainda, um tempo médio de consumo de 24 anos, apontando a adolescência, não só como período de início do consumo, mas também, como período de abuso da substância psicotrópica, o qual se deu por volta dos 18 anos. Diante disso, acredita-se ser importante investir no desenvolvimento de estratégias de atenção à saúde direcionadas a esta população, através do desenvolvimento de ações de prevenção e tratamento adequadas às suas necessidades.

Frente a estes resultados, nota-se a necessidade de um realinhamento discursivo entre a realidade do consumo de drogas e as políticas e intervenções direcionadas a estes usuários. Considera-se, ainda, a necessidade de realização de pesquisas que busquem ampliar o conhecimento acerca do fenômeno das drogas a fim de auxiliar no desenvolvimento de práticas que contemplem as singularidades desses sujeitos.

\section{Referências Bibliográficas}

1. Organização Mundial da Saúde. Organização Pan-Americana da Saúde (OPAS). Relatório Sobre a Saúde no Mundo. Saúde Mental: nova concepção, nova esperança. Genebra; 2001.

2. Rodrigues LBF. Drogas e cárcere: a repressão às drogas e o aumento da população penitenciária brasileira. In: Metaal P., Youngers AC., editores. Sistemas sobrecargados: leyes de drogas y cárceles en América Latina. Amsterdam/ Washington: TNI/WOLA; 2010. 30-40. 
3. Machado AR, Miranda PSC. Fragmentos da história da atenção à saúde para usuários de álcool e outras drogas no Brasil: da Justiça à Saúde Pública. História, Ciências, Saúde - Manguinhos. 2007; 14(3): 801-821.

4. Brasil, Ministério da Saúde. A Política do Ministério da Saúde para atenção integral a usuários de álcool e outras drogas. Ministério da Saúde - Secretaria de Atenção a Saúde. 2.ed. Brasília: Ministério da Saúde, 2004.

5. Bucher R, Oliveira SRM. O discurso do "combate às drogas" e suas ideologias. Revista Saúde Pública. 1994; 28 (2): 137-145.

6. Reis FC, Silva AA. Adolescência: consumo de álcool e outras drogas. Revista Enfermagem Integrada. 2009; 2(1): 112-125.

7. Souza J, Kantorski LP. Embasamento Político das Concepções e Práticas Referentes às Drogas no Brasil. Revista Eletrônica Saúde Mental álcool e Drogas. 2007; 3(2): 1-17.

8. Pelisoli CL, Moreira AK. Caracterização epidemiológica dos usuários do Centro de Atenção Psicossocial Casa Aberta. Revista Psiquiatria. 2005; 27 (3): 270-277.

9. Formiga LT, Santos RCS, Dumcke TS, Araujo RB. Comparação do Perfil de Dependentes Químicos Internados em uma Unidade de Dependência Química de Porto Alegre/RS em 2002 e 2006. 2009; 29 (2):120-126.

10. Brasil, Ministério da Saúde. Conselho Nacional de Saúde. Resolução n ${ }^{\circ} 466$, de 12 de dezembro de 2012. Aprova as diretrizes e normas regulamentadoras de pesquisa envolvendo seres humanos. Brasília, 2012.

11. Laranjeira R, Pinsky I, Zaleski M, Caetano R, organizadores. I Levantamento Nacional sobre os padrões de consumo de álcool na população brasileira. Brasília: Secretaria Nacional Antidrogas; 2007.

12. Noto AR, Baptista MC, Faria ST, Nappo SA, Galduróz JCF, Carlini EA. Drogas e saúde na imprensa brasileira: uma análise de artigos publicados em jornais e revistas. Cadernos de Saúde Pública. 2003; 19(1): 69-79.

13. Acselrad G. Educação para a autonomia: construindo um discurso democrático sobre as drogas. In: Acselrad G. Avessos do Prazer: Drogas, AIDS e direitos humanos. 2a ed. Rio de Janeiro: FIOCRUZ; 2005. p.183-211.

14. Mesquita F. Políticas públicas de drogas: A construção de um caminho democrático e humanitário para o Brasil. 2004.

15. Ronzani TM, Furtado EF. Estigma social sobre o uso de álcool. Jornal Brasileiro Psiquiatria. 2010; 59(4): 326-333.

16. Aberastury A, Knobel M. Adolescência normal: um enfoque psicanalítico. Porto Alegre: Artmed, 2008.

17. Macrae E. Aspectos socioculturais do uso de drogas e políticas de redução de danos. Núcleo de Estudos Interdisciplinares sobre psicoativos. 2007. http://siteantigo.neip.info/textos_pesquisadores.htm.

18. Schenker M, Minayo MCS. A importância da família no tratamento do uso abusivo de drogas: uma revisão da literatura. Cadernos de Saúde Pública. 2004; 20(3): 649-659.

19. Carlini EA, Galduróz JC, Noto AR, Carlini CM, Oliveira LG, Nappo AS, et al. II levantamento domiciliar sobre o uso de drogas psicotrópicas no Brasil: estudo envolvendo as 108 maiores cidades do país - 2005. São Paulo: Páginas \& Letras, 2007.

20. Pratta EMM, Santos MA. Levantamento dos motivos e dos responsáveis pelo primeiro contato de adolescentes do ensino médio com substâncias psicoativas. Revista Eletrônica Saúde Mental Álcool Drogas. 2006; 2(2).

21. Moura YG, Silva EA, Noto AR. Redes sociais no contexto de uso de drogas entre crianças e adolescentes em situação de rua. Psicologia em Pesquisa. 2009; 3(01): 31-46.

\section{Natália Dalla Côrte Cantarelli}

Endereço para correspondência - Rua Visconde de Pelotas, 374, apto 508. Bairro Centro, CEP 97010-440, Santa Maria, RS, Brasil.

E-mail: ndallacortecantarelli@gmail.com

Currículo Lattes: http://lattes.cnpq.br/2830146925798022

Elsa K. da Rosa Marchesan - elsakarsburg@hotmail.com

Marion Caroline do Amaral - marion.1984@hotmail.com

Jadir Camargo Lemos - jadir.lemos@gmail.com

Recebido em 30 de janeiro de 2014.

Publicado em 13 de março de 2014. 